Onderzoekcentrum voor Bosbouw, Bosbedrijfsvoerin en Bospolitick - R.U.G, Direkteur - Diensthoofd, Prof. dr. ir. M. VAN MIEGROET

\title{
LE FORESTIER ET LES PROBLEMES DE L'ENVIRONNEMENT
}

\author{
par
}

\section{Van Miegroet}

\section{Le point de départ}

\subsection{Le but de la sylviculture}

Le but de la sylviculture est l'optimalisation durable de l'utilité de la forêt. Cette utilité est soumise à une variation qualitative et quantitative dans l'espace et le temps, mais correspond également à une relation durable entre l'être humain et la forêt.

Cette relation se caractérise par des exigences liées à l'espace et au temps ainsi que par une fonction forestièrc générale de caractère durable :

$1^{\circ} \mathrm{La}$ préservation primordiale de la forêt dans les sites requis afin d'assurer de manière permanente son utilité tout en tenant compte des mutations sociales et des exigences qui évoluent rapidement.

$2^{\circ}$ La conservation d'une surface boisée suffisante, déterminée par les besoins spécifiques d'une part et par la qualité des forêts de l'autre.

$3^{\circ} \mathrm{La}$ stimulation de la polyvalence fonctionnelle de la forêt.

Cette triple mission concerne dans le fond la population du monde tout entier, mais actuellement elle se limite encore uniquement au niveau de la planification nationale. A ce stade, elle se réalise d'ailleurs plus facilement au fur et à mesure qu'apparait un soici plus grand sur un plan plus réduit.

\subsection{La pression exercée sur la forêt}

Le taux de boisement du globe est descendu jusqu'à $29 \%$, c.à.d. qu'on a atteint un seuil (Budowski : „period of awareness") où l'on commence à s'inquiéter de la qualité et de la quantité 
disponibles de ce qu'on considérait auparavant comme un bien naturel inépuisable.

C'est pourquoi la plupart des pays industriels à forte densité démographique ne sont plus en mesure d'assurer leurs besoins en bois par leur propre production. A cause de l'industrialisation à l'outrance, de l'agriculture intensive et de la construction immobilière, les surfaces boisées ne sont plus assez nombreuses pour satisfaire tant du point de vue qualitatif que quantitatif aux besoins élémentaires d'ordre social, hygiénique et récréatif.

Cette pression croissante qui peut mener à la destruction de la forêt à défaut de mesures adéquates, a une triple cause:

$1^{\circ}$ L'accroissement de la population $\rightarrow$ diminution de la surface boisée par habitant (p.c.)

$2^{\circ}$ L'augmentation du niveau de vie matérielle :

$\longrightarrow$ besoin accru d'espace (construction de routes, de maisons, industric)

$\longrightarrow$ augmentation de la consommation individuelle et collective en bois = atteinte à l'intégrité de la forêt allant jusqu'à la surexploitation et le remplacement de la forêt par la plantation artificielle dans des conditions douteuses.

$\rightarrow$ un besoin plus fréquent de la présence physique de la forĉt (fonction sociale) $=$ atteinte qualitative à l'intégrité de la forêt.

$3^{\circ}$ La mobilité croissante de la population $\longrightarrow$ intensification de l'impact humain sur la forêt.

Cette situation exige que dans certains cas un choix soit opéré entre les différentes fonctions de la forêt ou que celles-ci se trouvent face à face dans une position antagoniste.

La mobilité croissante de la main d'ceuvre et du capital intensifient et élargissent cet impact: une des conséquences en est l'appauvrissement et même la disparition de la forêt dans des régions isolées, où commencent à opérér des entreprises industrielles qui ne disposent plus assez de surfaces boisées dans leur propres pays ou qui ne peuvent pas y exploiter la forêt impunément.

Ces pratiques, qui se caractérisent en général par une mécanisation poussée et par une rationalisation de l'exploitation des forêts, peuvent avoir de graves conséquences:

a. La détériorisation rapide des richesses économiques et l'atteinte à la force de production naturelle des régions touchées. 
b. La destruction de larges zones naturelles à conserver en principe.

c. La modification profonde dans l'équilibre du milieu de vie, modification qui contient en puissance une menace à l'échelle mondiale.

\section{1,3. Options fondamentales}

L'évolution de l'utilisation de la forêt se caractérise par le déplacement des centres de gravité et par la polyvalence croissante de la fonction même de la forêt.

Il est vrai que dan les pays industrialisés à haute densité démographique la production de bois est encore considérée comme une fonction primaire, mais on constate quand même un intérêt croissant pour la signification sociale et hygiénique de la forêt. $\mathrm{La}$ sylviculture participe de ce fait à la transition vers le secteur économique tertiaire (la prestation de service).

Parmi les causes principales, on peut citer les relations sociales modifiées par la concentration de la population et par l'urbanisation de la campagne, ainsi que le progrès technologique et l'industrialisation accélérée qui représentent pour l'être humain un danger pour son environnement.

Le fait que l'on ne croit plus que les richesses naturelles soient inépuisables, qu'on doute de la stabilité bio-écologique et de la qualité de l'environnement sont à la base d'actions collectives en vue de: rieure;

a. protéger l'environnement contre toute dégradation ulté-

b. conserver de régions précieuses du point de vue scientifique, culturel et esthétique;

c. constituer et sauvegarder des zones de récupération à caractère récréatif;

d. assurer la production des matières vitales de base (cau pure, air riche en oxigène, etc.)

La reconnaisance de l'importance de la forêt pour la fonction générale de l'environnement (régulation du climat, lutte contre l'érosion, alimentation en eau = fonction économique indirecte) et en tant qu'élément constituant immédiat de l'environnement (zone récréative à l'abri de la pollution, zone de silence $=$ fonction sociale) diminue la signification de la fonction économique directe surtout dans les pays industrialisés manquant de bois. Pour leur 
approvisionnement en bois ces pays comptent de plus en plus sur des importations.

Dans les régions tropicales et subtropicales non-industrialisées dont les réserves en bois semblent inépuisables, c'est la fonction de surface et de production d'une matière première qui domine encore. L'exploitation de ces réserves constitue en quelque sorte le point de départ pour un accroissement rapide du niveau de vic de la population autochtone puisqu'elle y favorise le commerce et l'industrialisation. Pourtant une exploitation peu judicieuse et surtout trop rapide qui met en œuvre des moyens techniques puissants, le remplacement de la forêt naturelle par des plantations forestières industrielles peuvent ultérieurement avoir de graves conséquences sociales et politiques.

Ces pratiques peuvent être à la base de problèmes concernant l'environnement, dont l'ampleur et les conséquences concernent la population du monde entier.

Une exploitation sauvage peut mener à une dégradation massive de la forêt et à l'élimination de sa fonction économique.

De plus en plus, la sylviculture actuelle se considère ainsi placée devant l'alternative d'un objectif social et indirectement économique et la production d'unc matière première qui se raréfie et qu'une industrie de transformation influente revendique avec insistance.

Entre le motif économique et le motif social le choix n'est toutefois guère possible: tous deux correspondent à des besoins vitaux et à des exigences qui se manifestent simultanément. Dans des circonstances favorables, il est licite de poursuivre la satisfaction simultanée des besoins sociaux et économiques dans une seule et même aire boisée à condition d'adapter l'aménagement à des besoins multiples.

Dans une mesure croissante il s'agira toutefois de poursuivre une division fonctionnelle de la surface boisée en se basant sur ces motifs primordiaux de l'utilisation de la forêt :

- Ia préservation de la forêt $=$ utilité économique indirecte (protection générale de l'environnement);

- utilisation sociale de la forêt $=$ utilité sociale (protection immédiate de l'environnement);

- production de bois $=$ utilité économique directe (utilité sociale indirecte). 
Cette division fonctionnelle de l'espace doit toutefois se limiter à un niveau assez restreint: sur le plan international une telle conception ne pourrait s'appuyer que sur une division injuste de la richesse et de la puissance; sur le plan national, elle est incompatíble avec l'égalité des droits de tous les citoyens.

Lors de la réalisation d'une division fonctionnelle de l'aire boisée, l'on peut se baser sur trois types fondamentaux de forêts :

\section{Les forêts protégées}

Il faut assurer leur sauvegarde à tout prix, en raison de leur effet bénéfique, de leur utilité économique indirecte et de leur importance pour l'environnement.

Les véritables peuplements d'abri, les réserves forestières, les forêts dans les réserves naturelles, les forêts à fonction spécifique dans le domaine de l'alimentation en eau, la régulation du climat, l'approvisionnement en oxygène appartiennent à cette catégorie.

\section{Les forêts à fonction sociale prédominante}

Ge sont les forêts à vocation récréative mais qui n'excluent pas une production de bois axée sur la qualité et sur des essences de choix.

\section{Les forêts économiques}

Elles sont entic̀rement axćes sur la production d'une matière première industrielle, y compris une large part des peuplements de conifères homogènes, implantés artificiellement.

La détermination excate de ces formes primaires d'utilisation est d'importance capitale en vue de l'accroissement optimal de leur efficacité.

La division fonctionnelle des espaces est basée sur la reconnaissance de besoins multiples qui ne peuvent pas toujours être satisfaits simultanément et au même endroit.

Elle se base également sur la reconnaissance de la valeur relative des fonctions forestières et du caractère dynamique de l'évolution sociale qui cause des modifications qualitatives et quantitatives des besoins.

\section{Le rôle de la sylviculture dans la protection de l'environ- nement}

La forêt assume plusieurs fonctions, que Hasel (Waldwirtschaft und Umwelt, Hamburg, u. Berlin 1971) définit comme suit : 
1 Activité de surface

2. Effet protecteur: a. Climat

b. Pureté de l'air

c. Protection contre le bruit

d. Protection contre les phénomènes de radiation

c. Alimentation en eau

f. Préservation de la fertilité du sol

3. Rôle récréatif

4. Production de matière première

5. Source de revenus

6. Constitution de réserves

7. Source d'emplois

8. Accroissement de l'avoir

9. Chasse

10. Fonction culturelle

Dans le domaine de la préservation de l'environnement, ce sont les fonctions directement liées à la présence physique de la forêt, qui sont importantes; c'est pourquoi la stimulation de ces fonctions doit être considérée comme un objectif primordial.

La sylviculture peut en outre intervenir utilement dans la solution de problèmes concernant la protection de la nature, la préservation des sites et l'aménagement du territoire.

2.I. Les missions primaires de la sylviculture dans la protection de l'environnement

\subsubsection{La fonction de surface}

L'importance de la présence physique de la forêt crốt au fur et à mesure que la surface boisée disparaît, que la population augmente et que l'utilisation de la forêt devient plus intense. Lorsque la pression exercée sur la forêt augmente démesurément, l'on atteint fatalement un seuil où il devient difficile, voire impossible de satisfaire tous les besoins.

La satisfaction des besoins sociaux et hygiéniques pose alors les plus grands problèmes, puisqu'il est provisoirement encore possible de pallier au manque de bois en tant que matière première par l'importation.

La surface boisée minimum qui permette de couvrir les besoins en bois par une production en propre peut être calculée sur base de l'indice de consommation et de la productivité des forêts. II 
est plus difficile de définir la surface boisée dont il faudra disposer pour satisfaire aux besoins sociaux de l'avenir.

Selon Hasel toutes les fonctions forestières peuvent être optimalement assumées lorsque le taux de boisement est de $50 \%$ pour les régions fort montagneuses et de $30 \%$ pour la plaine,

Un taux de $25 \%$ est inquiétant et sous les $20 \%$ une grave rupture des fonctions forestières semble inévitable.

D'après ces normes la situation sur le plan mondial (29\%) n'est guère favorable; elle est mềme relativement défavorable en Afrique $(24 \%)$ en Asie (19\%) et en Océanie (11\%). En Europe des problèmes se posent pour les pays suivants :

\begin{tabular}{|c|c|c|c|c|c|}
\hline \multicolumn{2}{|c|}{ Situation marginale } & \multicolumn{2}{|c|}{ Situation défavorable } & \multicolumn{2}{|c|}{ Situation critique } \\
\hline $\begin{array}{l}\text { Pologne } \\
\text { R.D.A. } \\
\text { Suisse } \\
\text { Italie } \\
\text { France }\end{array}$ & $\begin{array}{l}25 \% \\
25 \% \\
25 \% \\
21 \% \\
29 \%\end{array}$ & $\begin{array}{l}\text { Belgique } \\
\text { Grèce } \\
\text { Hongrie }\end{array}$ & $\begin{array}{l}19 \% \\
16 \% \\
14 \%\end{array}$ & $\begin{array}{l}\text { Danemark } \\
\text { Pays-Bas } \\
\text { U.K. } \\
\text { Eyre }\end{array}$ & $\begin{array}{l}9 \% \\
7 \% \\
7 \% \\
3 \%\end{array}$ \\
\hline
\end{tabular}

Lors de l'établissement de la surface boisée minimum requise, il faut en premier lieu tenir compte de la densité de la population qui s'exprime dans la surface boisée disponible par habitant (p.c.). De ce point de vue, l'explosion démographique et l'industrialisation ont aggravé la situation : c'est ainsi qu'en R.F.A. la surface boiséc par habitant a baissé de 0,32 ha en 1878 jusqu'à 0,13 ha en 1961 en dépit d'un accroissement de la surface boisée absolue. Dans beaucoup de pays européens la situation est marginale:

\begin{tabular}{|c|c|c|c|c|c|}
\hline \multicolumn{2}{|c|}{$0,15-0,20$ ha p.c. } & \multicolumn{2}{|c|}{$0,10-0,15$ ha p.c. } & \multicolumn{2}{|c|}{$<0,10$ ha p.c. } \\
\hline $\begin{array}{l}\text { France } \\
\text { Pologne } \\
\text { Luxembourg } \\
\text { Suisse } \\
\text { R.D.A. } \\
\text { Grèce }\end{array}$ & $\begin{array}{l}0,20 \\
0,20 \\
0,20 \\
0,17 \\
0,16 \\
0,20\end{array}$ & $\begin{array}{l}\text { R.F.A. } \\
\text { Hongrie } \\
\text { Italie }\end{array}$ & $\begin{array}{l}0,13 \\
0,13 \\
0,12\end{array}$ & $\begin{array}{l}\text { Danemark } \\
\text { Eyre } \\
\text { Belgique } \\
\text { U.K. } \\
\text { Pays-Bas }\end{array}$ & $\begin{array}{l}0,08 \\
0,07 \\
0,06 \\
0,03 \\
0,02\end{array}$ \\
\hline
\end{tabular}

Il s’agit donc de déterminer la surface boisée nécessaire par habitant pour en déduire la surface boisée absolue qu'il faut atteindre. 
Il est également nécessaire d'assurer une répartition efficace des forêts pour qu'elles puissent atteindre à un rendement social et à une influence sur l'environnement aussi favorables que possible.

Les boisement antérieurs et la préservation des forêts sur base de normes économiques ne correspondent plus à tous les besoins actuels : c'est dans les zones industrielles à forte population que la forêt fait le plus défaut et c'est pourtant dans ces régions que son effet social est le plus élevé. Une répartition judicieuse des surfaces boisées sur chaque unité géographique d'un état, évite des tensions et aussi une pression inégalement exercée sur la forêt, tant du point de vue quantitatil que qualitatif.

Un tel principe n'est pas uniquement valable pour de grands pays, mais aussi pour les petits, comme le prouve la situation en Belgique $\left(30.000 \mathrm{~km}^{2}\right)$ :

\begin{tabular}{|l|c|c|c|c|}
\hline \multicolumn{1}{|c|}{ Province } & $\begin{array}{c}\text { Habitants } \\
\mathrm{km}^{2}\end{array}$ & $\begin{array}{c}\text { Taux de } \\
\text { boisement }\end{array}$ & $\begin{array}{c}\text { Surface forêt } \\
\text { p.c. }\left(\mathrm{m}^{2}\right)\end{array}$ & $\begin{array}{c}\text { Forêt d'état } \\
\text { p.c. }\left(\mathrm{m}^{2}\right)\end{array}$ \\
\hline Luxembourg & 50 & 44,4 & 8.935 & 721 \\
Namur & 105 & 31,8 & 3.037 & 197 \\
Liège & 258 & 27,9 & 1.083 & 246 \\
Hainaut & 358 & 13,6 & 380 & 22 \\
Anvers & 535 & 13,7 & 256 & 18 \\
Limbourg & 270 & 15,0 & 556 & 29 \\
Brabant & 660 & 9,7 & 147 & 36 \\
Fl. Or. & 440 & 4,1 & 93 & 3 \\
Fl. Occ. & 325 & 2,6 & 79 & 8 \\
Belgique & 317 & 19,7 & 622 & 67 \\
\hline
\end{tabular}

Une répartition inégale crée des tensions entre la ville et la campagne, entre les besoins économiques et les exigences sociales.

Dans les régions peu boisées il faut en outre combattre le morcellement de la forêt, d'ailleurs très fréquent dans ces zones. Con trairement aux surfaces en friche une forêt ne peut être divisée sans subir une perte en valeur et d'efficacité (Leibundgut). Un aménagement forestier normal sous-entend en effet une surface boisée minimum dont la superficie doit toutefois être assez importante. Le morcellement de la forêt augmente aussi l'impact des facteurs sylvidestructeurs (châblis, nécrosie, dommages de neige) et diminue le rendement fonctionnel, surtout dans les régions où la fonction de la forêt est déjà marginale.

Lors de l'élaboration des mesures indispensables les différents types de propriété forestière exerceront une influence déterminante. 
Dans les pays où l'état possède beaucoup de forêts, il est en principe facile de décréter des mesures légales généralisées. Là où la propriété forestière est collective (forêt ou bois communal, bois corporatif) des solutions régionales peuvent être trouvées rapidement. Ce n'est que quand la forêt est une possession privée qu'il est plus difficile de prendre des mesures d'intérêt général sans porter atteinte au libre exercice du droit de propriété privée.

En Europe il est donc difficile d'envisager dés mesures générales dans les pays où l'état ne possède que peu de forêts comme en Italie (4\%), en Suisse (5\%), en Belgique (12\%), en France $(14 \%)$ et en Autriche (17\%).

Il faut tenir compte des intérêts privés un peu partout en Europe, mais plus spécialement en Belgique (52\%), au Luxembourg $(57 \%)$, aux Pays Bas $(58 \%$ ), en Italie $(61 \%)$, dans le Royaume Uni $(63 \%)$, en France $(64 \%)$, en Espagne (69\%), en Finlande $(70 \%)$, en Suède $(75 \%)$, en Norvège $(80 \%)$ et en Autriche (80\%).

Des problèmes particuliers dans le domaine des relations de propriété se posent à la périphéric des grandes villes où la forêt privéc est forcément utilisée par le public à des fins récréatives lorsque la forêt publique disponible est insuffisante ou fait défaut.

\subsubsection{La fonction protectrice}

L'influence bienfaisante générale de la forêt (protection contre l'érosion, régulation du climat, alimentation en eau, lutte contre le bruit) est suffisamment connue. Deux aspects méritent une attention particulière, la prévention de la pollution atmosphérique et l'alimentation en eau.

\section{La pureté de l'air}

Dans la plupart des régions le rôle joué par la forêt en tant que producteur d'oxygène est limité. Keller a calculé que pour la Suisse 1 ha de forêt produit une quantité d'oxygène égale à la consommation de 10 habitants (250kg/an). Ceci ne prouve pas du tout l'existence d'une relation directe : en effet, la production d'oxygène est surtout l'ouvre des océans $(70 \%)$ et des forêts tropicales toujours vertes. La consommation humaine d'oxygène ne constitue en outre qu'une infirme fraction de la consommation totale et les réserves en oxygène dans l'atmosphère sont fort élevées $\left(2.000 \mathrm{~kg} / \mathrm{m}^{2}\right)$,

La forêt ne sert pas non plus de filtre utile aux gaz nocifs ( $\mathrm{SO}_{2}$, les combinaisons de chlore et de fluor, les hydrocarbures) : la capacité d'absorption est réduite ce qui provoque rapidement des dommages de fumée (plasmolyse, nécrose).

L'importance de la forêt réside dans sa fonction passive et 
dans sa grande capacité filtrante par rapport aux pollutions atmosphériques solides (Keller, Meldau, Jutzi).

$1^{\circ}$ La forêt est capable de fixer de grandes quantités de particules d'air (32 $\mathrm{T}$ par ha pour une pessière, $68 \mathrm{~T}$ pour une hêtraie).

$2^{\circ}$ La couronne-filtre se régénère par la pluie.

$3^{\circ}$ L'action filtrante des essences feuillues est plus grande que celle des conifères (Keller).

\begin{tabular}{|l|c|c|c|}
\hline Essence & $\begin{array}{c}\text { Interception mg. } \\
\text { de poussière par g. } \\
\text { de poids à scc }\end{array}$ & Poids feuille kg/ha & $\begin{array}{c}\text { Interception } \\
\text { poussièrc } \\
\text { kg/ha }\end{array}$ \\
\hline Hêtre & 70 & 4.000 & 280 \\
Chêne & 90 & 6.000 & 540 \\
Epicéa & 30 & 14.000 & 420 \\
\hline
\end{tabular}

\section{Alimentation en eau}

L'approvisionnement en eau - que ce soit en quantité ou en qualité - devient un problème primordial pour le monde industrialisé. Les réserves en eau souterraine ne suffisent déjà plus depuis longtemps dans maintes régions et l'eau de surface est lourdement polluée. En Belgique le niveau maximum de consommation d'eau souterraine a déjà été atteint en 1965 (1.100 millions de $\mathrm{m}^{3} / \mathrm{an}$ ); il a d'ailleurs fallu y ajouter 400 millions de $\mathrm{m}^{3} \mathrm{~d}^{\prime}$ eau de surface depuis 1968.

Ces quantités d'eau de surface s'élèveront jusqu'à 900 millions en 1980 et 2.500 à l'an 2.000 .

La forêt devra être utilisée comme collectrice d'eau et comme filtre naturel, surtout dans la plaine où l'eau ne s'accumule pas dans les gletschers. Les régions agraires n'entrent plus en ligne de compte en raison de leur influence polluante (engrais chimiques, déchets, pesticides).

Voets (Université de l'Etat à Gand) a calculé que l'influence polluante sur l'eau de surface exercée en Belgique par le cheptel représente 41,000.000 d'équivalences - habitant et que l'agriculture décharge 49.352 $\mathrm{T}$ d'azote dans l'eau de surface tandis que $43.000 \mathrm{~T}$ proviennent seulement de zones habitées (non compris la pollution industrielle).

\subsubsection{La fonction récréative}

La récréation forestière correspond à une exigence sociale 
réelle. L'existence de forêts récréatives diminue la pression de la population sur la forêt économique. C'est pourquoi il faut aménager une quantité suffisante de forêts récréatives, soigner pour une bonne répartition géographique et pour leur implantation à la périphérie des grandes zones urbaines et industrielles. En n'oubliant pas le principe qu'une forêt ne supporte que la présence simultanée de 15 personnes par ha et que les besoins en récréation peuvent s'ćlever les jours de pointe jusqu'à 15 à $20 \%$ de la population, la formule suivante est valable:

$\mathrm{S}=\frac{\mathrm{B}}{75}$ jusquía $\frac{\mathrm{B}}{100}$ où $\mathrm{S}=$ surface boisée récréative et $\mathrm{B}=$ indice de population (nombre d'habitants)

Elle s'applique à la plus petite unité géographique.

Les qualités récréatives de la forêt sont déterminées par les facteurs suivants :

- la structure de la lisière : elle détermine l'attrait et le type d'activités.

- la combinaison des essences : le contraste, la polyvalence et la variété sont des qualités positives.

- le mode de traitement: la répartition des classes d'âge, les types d'exploitation et les procédés de rajeunissements ont importants.

Des forêts saines et stables, comportant de nombreuses essences excercent le plus grand attrait.

La forêt de récréation est compatible avec la production économique, à condition que soient définies la valeur relative des fonctions sociales et économiques et qu'une division fonctionnelle de l'espace soit entreprise sur base par exemple de la typologie suivante :

le parc dans l'agglomération

le parc forestier à la périphérie des zones habitées

la forêt de récréation dans les endroits facilement accessibles la forêt économique dans les endroits plus éloignés

la forêt-réserve à protéger, à n'importe quel prix.

L'organisation de la forêt d'agrément crée de nouvelles responsabilités dans le domaine

de la recherche sociologique (examen des motivations, typologie de la récréation forestière, l'évolution des besoins récréatifs)

de la technique de récréation (formes, infrastructures indispensables) 
du traitement (structure des peuplements, répartition des classes d'âge, orientation de la production économique).

\subsubsection{La fonction de réserve}

Par sa longévité et sa longue occupation des stations, la forêt opère la stabilisation de l'utilisation des terres.

Une préservation systématique des forêts dans les zones tropicales et subtropicales ainsi que le boisement immédiat des espaces libérés dans les zones industrielles et agraires extériorisent la propriété de la forêt à constituer une réserve de sols.

Ceci permet d'atteindre un triple objectif :

$1^{\circ}$ Eviter la spéculation immobilière et inciter à la planification en ce qui concerne l'utilisation non fixée de terrains.

$2^{\circ}$ Constitution d'une réserve de terrains qui peuvent être libérés plus tard en cas de besoin.

$3^{\circ}$ Garantir les effets bienfaisants indispensables.

Par sa fonction de réserve de sols la forêt contribue à la stabilisation des structures sociales et à la conservation des possibilités futures de développement.

Deux conditions doivent toutefois être remplies :

La définition de la surface boiséc absolue c.à.d. la superficie forestière absolue en deçà de laquelle l'on nuit aux intérêts économiques et sociaux.

Assurer autant que possible le caractère réversible de l'échange sols.

Il faut en effet qu'un nombre suffisant d'arguments de nature durable soient présents pour procéder à la destruction irréversible de la forêt (construction de routes, implantations industrielles, constructions immobilières, etc.). Des échanges réversibles ont continuellement eu lieu entre l'agriculture et la sylviculture. Dans le cadre de l'évolution actuelle, caractérisée par la concentration de l'agriculture, l'élimination des entreprises marginales (le plan Mansholt d'assainissement prévoit pour la C.E.E. le transfert de $5,000.000$ ha de surfaces agricoles vers la sylviculture et les accommodations de récréation), l'industrialisation de l'agriculture (entreprises bio-industrielles), la sylviculture doit être en mesure d'occuper les zones agricoles libérées afin d'élargir la fonction sociale de la forêt.

\subsection{Les missions secondaires de la sylviculture}

Par la force des choses, la sylviculture entre en contact avec 
d'autres activités humaines qui peuvent faire valoir des droits sur l'occupation des sols. Jadis cela a souvent mené à une espèce de compétition basée sur des malentendus savamment entretenus, sur l'incertitude quant à l'évolution sociale et sur le rôle de l'homme dans une société dynamiqué.

L'acceptation de la position centrale de l'être humain, une conception élargie de la dynamique et de l'évaluation objective des motifs et des types d'utilisation des sols, fait comprendre que les différentes activités humaines doivent se compléter mutuellement. L'ampleur croissante de la connaissance acquise dans de nombreux domaines fait naître le besoin de coopération et de pénétration interdisciplinaire. Cette coopération doit s'appuyer sur le pluralisme idéologique naissant, sur l'importance de l'information réciproque et sur la nécessité d'analyser sous tous ses angles le problème posé. C'est ainsi que la sylviculture peut sortir de son isolement relatif et contribuer à la solution de problèmes qui n'appartiennent pas directement à son terrain d'action.

\section{2..21. Protection de la nature}

Entre la sylviculture et la protection de la nature ont existé des malentendus qui sont la conséquence d'une conception unilatérale des missions mutuelles. Le forestier peut, en effet, rendre de nombreux services dans le domaine de la protection de la nature :

Il administre un bien qui occupe un territoire important et à l'intérieur duquel il peut souvent réaliser la stabilité biologique et l'équilibre écologique par le maintien de la diversité et le mélange des essences, choisies de préférence parmi les espèces autochtones.

Son activité repose sur la compréhension de la dynamique naturelle de l'équilibre et est basée sur l'observation de la nature et l'interprétation des phénomènes biologiques.

Il considère la forêt comme une communauté vivante complexe qui possède sa propre structure, son propre rythme de vie et son caractère biocénétique, dont la flore comme la faune sont des composantes essentielles.

C'est pourquoi la sylviculture moderne estime que la préservation et la réalisation de la richesse biologique et de la variété des espèces constituent un objectif capital.

La création de réserves forestières fermées - ce qui permet une évolution libre - est une manifestation de l'intention de rendre possible l'observation de la nature et l'étude des lois fondamentales qui dominent la dynamique de croissance des peuplements.

En établissant de larges rideaux forestiers périphériques 
autour des zones naturelles qu'il faut préserver, le sylviculteur contribue à la création de zones-tampons psychologiques et physiques que le public peut accepter.

La sylviculture peut jouer le rôle de modérateur entre les exigences de la protection absolue de la nature et les besoins en air frais d'un nombre toujours croissant de personncs. L'établissement de zones de récréation et de forêts-promenade ne diminue pas seulement la pression humaine sur la forêt économique mais aussi sur les réserves naturelles à préserver.

\subsubsection{Structure du paysage}

La valeur d'un paysage est déterminée par sa richesse plastique, sa diversité et sa stabilité. Il s'agit de l'aboutissement d'une évolution culturelle et historique et non pas d'une création artificielle inspirée par des considérations esthétiques. La structure harmonieuse d'un paysage provient de l'implantation correcte de formes stables d'utilisation des sols. Par sa forme et par son ampleur la forêt est un élément essentiel du paysage.

Elle donne au paysage sa stabilité en dépit du grand nombre de variétés qu'elle peut offrir, ce qui s'exprime d'ailleurs dans plusieurs domaines:

composition : choix des essences, alternance des groupes d'âge. variation spatiale: mélange d'essences et de classes d'âge, alternance d'espaces boisés et de clairic̀res.

apparence: choix du mode de traitement et technique d'exploitation.

évolution dynamique: choix du mode et du rythme de rajeunissement.

La mission normale de la sylviculture consiste à dominer ces éléments.

Le fait d'être axé trop unilatéralement sur les buts économiques de la sylviculture peut entraîner la rupture de l'équilibre du paysage et s'accompagner de sa dépréciation provisoire ou définitive.

L'accroissement de la récréation de lisière, conséquence inévitable du trafic automobile croissant et de la démocratisation du tourisme, a considérablement augmenté la signification de la forêt en tant que refuge ou aire de repos provisoire le long des axes routiers. 
La forêt s'insère parfaitement dans l'élargissement de l'échelle du paysage moderne par suite de l'expansion industrielle, de l'extension des zones habitées, de la constitution d'unités d'exploitation agricole plus grandes et de l'accélération du trafic. Il s'agit en effet d'un type d'utilisation des sols qui ne s'exprime pleinement qu'au moment oú une surface de terrain d'une seule tenue peut être occupéc.

\subsubsection{Aménagement du territoire}

L'aménagement du territoire découle de la reconnaissance d'impératifs sociaux et économiques dus à la concentration de la population et à la nécessité de la création de zones habitables et industrielles équilibrées, valables et saines. Le rôle de la sylviculture dans une telle entreprise est corollaire de la multiplicité de ses fonctions ainsi que des exigences sociales et économiques auxquelles elle répond.

Il existe des raisons pertinentes pour inclure la sylviculture dans la planification et la prise de décisions dans le domaine de l'aménagement du territoire:

$1^{\circ}$ En raison de l'importance indéniable de la forêt, il faut tenir compte de l'avis du forestier à chaque niveau. Le rôle de la forêt devient plus effectif au fur et à mesure que son utilisation se situe à un niveau d'aménagement plus restreint.

$2^{\circ}$ Là où la forêt fait défaut, l'avis du forestier n'est pas moins important: l'absence de forêt peut être révélatrice d'un manque d'équilibre et peut diminuer la valeur fonctionnelle d'une région ou d'un paysage.

$3^{\circ} \mathrm{Il}$ faut également consulter le forestier sur l'utilisation future de la forêt, en cas de boisement et là où une exploitation définitive est envisagée, car il est en mesure d'évaluer concrètement les conséquences des décisions recommandées.

L'avis de la sylviculture est surtout souhaité lorsqu'il s'agit de formes d'utilisation des sols avec lesquelles elle entre en compétition.

Tel est par exemple le cas du remembrement des terres agricoles qui poursuit en principe l'amélioration de l'infrastructure agraire et un élargissement d'échelle mais qui mène fréquemment à un appauvrissement en substances biologiques sur de larges étendues.

Souvent le remembrement est à l'origine d'une pollution aggravée de l'eau et elle favorise l'érosion en faisant disparaitre le dernier arbre et le plus petit bosquet.

La mise en réserve d'une partie de la zone à restructurer 
(par. ex. $10 \%$ ) par un boisement approprié éliminerait la quantité des objections et contribuerait à rendre plus acceptable le procédé.

Un système analogue pourrait être mis en place lorsqu'il s'agit de lotissements de terrains à bâtir.

Lors de l'établissement des zones vertes à buts récréatif et hygiénique, le boisement offre également de grands avantages :

$1^{\circ}$ Les frais de plantation et d'entretien sont peu élevés.

$2^{\circ}$ L'occupation est rapide et il est possible d'adapter avec souplesse la forêt aux besoins en évolution.

$3^{\circ}$ Il constitue une base large pour une multitude d'activités.

$4^{\circ}$ La rentabilité économique n'est pas exclue.

\subsection{Conclusions}

La mission primaire de la sylviculture dans le cadre de la protection de la nature est la conséquence de la nature même de la forêt, élément important et stable du milieu de vie humain. L'influence qualitative et quantitative de la forêt cst grande à cause de la complexité de sa structure et. sa longévité.

La sylviculture doit veiller à ce que la surfacé forestière ne descende pas sous un seuil limite que détermineront les besoins économiques, sociaux et hygiéniques. On augmentera l'efficacité de la forêt grâce à une bonne répartition et à la lutte contre le morcellement; en aucun cas la fonction d'écran assuméc par la forêt ne saurait être mise en danger. La sylviculture peut aussi jouer un rol important en coopérant avec d'autres domaines (l'agriculture, l'aménagement du paysage, la planologie, l'économie, les techniques de culture, etc.). Une telle collaboration suppose toutefois une compréhension réciproque qui naîtra d'une efficace relation des intérêts, des objectifs et des possiblités, ainsi que de la pénétration interdisciplinaire dans le domaine de la recherche, de la formation, de la planification et de l'exécution.

L'orientation sociale de la sylviculture exige de ses représentants qu'il réfléchissent à leurs responsabilités envers la société et qu'ils y adaptent leurs conceptions, leurs méthodes et leurs procédures.

\section{Les adaptations nécessaires}

Le fait d'attribuer à la sylviculture des objectifs multiples et de lui faire assumer une responsabilité dans le domaine de la protection de l'environnement, exige un élargissement des con- 
ceptions sylviculturelles, une modification de profil professionel du forestier et aussi des adaptations dans bon nombre d'autres domaines,

\subsection{Le traitement des forêts}

La fonction sociale, récréative et hygiénique que certaines régions boisées doivent assumer, influence directement l'aménagement et le choix du type de traitement (élimination de la coupe rase, stimulation du mélange et du rajeunissement naturel).

Dans ce cas, tout comme aux endroits où l'action protectrice de la forêt est capitale, la préservation de la forêt, la stabilisation de sa structure et une occupation rapide des terrains en friche deviennent des objectifs primordiaux. Dans certains cas la production économique peut devenir secondaire; elle peut même disparaitre complètement dans les zones où la fonction sociale est considérée comme étant la plus importante. Une interpénétration des deux fonctions n'est d'ailleurs pas exclue à condition de prendre certaines précautions.

La perte d'une production de bois directe, sous forme d'une diminution de l'accroissement par suite de l'utilisation récréative fréquente de la forêt, d'une limitation des coupes, du choix approprié des essences ou d'une restriction de la liberté d'action technique, peut être compensée de plusieurs manières :

$1^{\circ}$ La valorisation de la fonction de prestation de service qui doit être considérée comme une promotion du secteur économique primaire vers le secteur tertiaire et pour laquelle il faut mettre au point des critères d'appréciation adéquats.

$2^{\circ}$ La réorientation de la production de bois qui doit être en harmonie avec les exigences de la récréation. Elle doit porter sur la production de la qualité, l'individualisation des soins culturaux et sur la concentration de l'accroissement dans les arbres d'élite et les essences de haute valeur.

$3^{\circ} \mathrm{La}$ commercialisation des produits forestiers secondaires (bois de chauffage, branches d'ornement, herbes, fruits, etc.) grâce à la prospection et à l'étude du marché.

Dans les pays en voie de développement, les fonctions forestières sociales et économiques ne sont guère développées et de ce fait l'importance de la forêt se situe surtout au niveau de la présence physique (action bienfaisante), de l'action de surface (réserve de sol) et de la formation d'une réserve de bois (réserve de matières premières).

Une organisation rationnelle de l'exploitation et une utilisation forestière judicieuse permettent d'éviter dans ces régions les erreurs qui sont à la base de la crise écologique que traversent les pays 
industrialisés. Une industrialisation trop rapide ne saurait se faire qu'aux dépens de la forêt; les profits immédiats seraient réduits par les investissements ultérieurs à faire en vue de la lutte contre l'érosion et de la régénération du sol. La conservation de la forêt est primaire et il devient même urgent de délimiter - même au niveau mondial - des réserves forestières et naturelles qui doivent être sauvegardées en vertu de leur valeur écologique.

$\mathrm{La}$ recherche technologique doit poursuivre la valorisation des essences autochtones; les plantations forestières industrielles ne sont pas souhaitées lorsqu'il faut qu'une destruction systématique des forêts autochtones les précède. De telles plantations sont toutefois souhaitables dans les régions non boisées et à la périphérie des villes à haute réserve de main d'œuvre.

\subsection{Enseignement et recherche}

Le caractère polyvalent de la sylviculture a mené à un isolement professionnel qui s'extériorise également au niveau de la recherche et de l'enseignement universitaire :

$1^{\circ}$ L'utilisation très rare dans la sylviculture des résultats des progrès scientifiques et technologiques.

$2^{\circ}$ De rares échanges d'expérience avec d'autres disciplines scientifiques.

$3^{\circ}$ L'attitude pragmatique du sylviculteur professionel qui est orienté vers la pratique de la gestion et considère avec trop de méfiance la recherche fondamentale.

$4^{\circ}$ Le caractère empirique d'une grande partie de la recherche en sylviculture et l'oubli des problèmes fondamentaux (phénoménologie de la croissance, recherches physiologiques et bio-chimiques, etc.)

$5^{\circ}$ La perte de contact avec la collectivité en raison d'une conception policière du rôle administratif et la fermeture des bois au public.

Pour sortir de cet isolement, il faut modifier le profil professionnel du sylviculteur; il doit s'adapter aux besoins de notre époque et avoir une vision large de l'avenir.

Il faut surtout accentuer la motivation sociale, agrandir la connaissance générale de la problématique de l'environnement et élargir la formation scientifique de base afin de donner à l'activité forestière - dont le caractère technique reste fondamental - une philosophie de base, axée sur l'intérêt commun.

La formation forestière doit de ce fait reposer sur une base scientifique solide (les sciences naturelles) et stimuler dès le début 
un intérêt social spécifique (économie, sociologie). Dès le stade initial l'étude doit être orientée vers un contact constant avec d'autres disciplines les plus diverses.

Il faut créer une recherche forestière spécifique et fondamentale dont les liens avec l'enseignement doivent être étroits afin de former des praticiens intéressés à la recherche.

Dans le domaine de l'enseignement et de la recherche - et même dans l'administration forestière directe - il faut, dans la mesure du possible, faire appel à des spécialistes d'autres disciplines. Avec le temps, cet échange pourrait prendre un caractère multilatéral, ce qui ne peut que favoriser la coopération.

Cettc coopération doit être préparée efficacement, déjà au stade de la formation du futur foresticr lui-même, en lui donnant l'occasion de suivre des cours en dehors de sa spécialité. Les cours les plus évidents sont les sciences naturelles avec la biologie et la biochimie comme disciplines de base. Une vision élargie de la mission de la sylviculture exige en outre un rapport accru de connaissance et d'information de la part du droit, des sciences sociales et économiques et même de la part de la médecine (science du travail, étude et influences du milieu).

Les responsables peuvent préparer cette réorientation par la mise en place de projets multidisciplinaires collectifs.

\subsection{L'administration forestière}

La forêt croît en importance au fur et à mesure que sa superficic relative et absolue diminue. En même temps les exigences quant à l'utilisation de la forêt augmentent en quantité et en fréquences, ce qui rend les tâches des organes administratifs responsables plus nombreuses et plus variées; à son tour, une telle orientation suppose une connaissance scientifique élargie et l'utilisation de techniques nouvelles.

La nouvelle ligne de conduite de la sylviculture doit premouvoir une utilisation polyvalente de la forêt et poursuivre un effet d'utilité maximal et durable. Une telle optique exige la mise en place d'une autre échelle de valeurs qui ne soit plus uniquement basée sur l'utilité économique et la rentabilité financière, tout comme l'adoption de mesures directes qui doivent s'extérioriser dans l'administration forestière.

Traditionnellement les administrations forestières publiques ont surtout poursuivi la rentabilité économique; sur le plan psychologique, technique et scientifique elles ne sont pas toujours à même d'opérer un choix nuancé entre les objectifs économiques et sociaux auxquels les confronte la société contemporaine. 
Elle subissent constamment des pressions en raison des nécessités de la protection de l'environnement comme des exigences de l'industrie du bois.

Une des solutions parfois envisagées est la fusion de l'administration forestière avec d'autres services publics; ceci favoriserait la coordination et diminuerait la concurrence, elle ne permettrait toutefois pas de modifier dans un avenir très proche les modalités et les principes de base de l'administration forestière.

Dans la plupart des administrations forestières publiques règne depuis le 19 e siècle le principe de la compétence territoriale qui est confiée à un fonctionnaire polyvalent.

Une telle situation n'est plus tenable si l'on tient compte :

- des nombreuses missions nouvelles de la sylviculture;

- de la compétence nécessaire dans dè nombreux domaines scientifiques et techniques;

- de l'opportunité de la formation permanente et du maintien de l'intétêt scientifique.

La compétence territoriale gêne en outre la réalisation de la coopération multidisciplinaire au niveau de l'administration.

Il paraít donc souhaitable de remplacer les principes de la responsabilité individuelle et de la compétence territoriale par une spécialisation professionnelle et par une administration collégiale.

Lors d'une telle administration collégiale (gestion de groupe) les divisions professionnelles suivantes peuvent être envisagées: boisement et reboisement, traitement des peuplements, exploitation de bois et vente, travaux techniques, administration, chasse, récréation forestière et protection de l'environnement, propriété forestière privée, aménagement du paysage, documentation et importation, pépinières, surveillance et poursuites judiciaires.

Les avantages techniques et administratifs d'une gestion collégiale sont évidents :

- engagement de personnel non-forestier sans nuire pour cela à l'ingénieur forestier; tratifs;

- conclusion rapide des problèmes techniques et adminis-

- rupture de l'isolement par l'intensification de l'impact des activités forestières qui pourront ainsi faire face à un champ plus large de tâches.

La transition vers une gestion collégiale peut provisoirement 
poser des problèmes en raison de l'absence de forestiers spécialisés et du manque d'intérêt de la part des autres disciplines.

L'organisation d'un recyclage permanent et d'une refonte énergique de l'enseignement universitaire doivent fournir à ces problèmes la solution définitive.

\section{Gonclusion finale}

Il faut que la sylviculture de l'avenir ait la possibilité d'accomplir la tâche élargie que la société moderne, en évolution dynamique et constante, exige d'elle.

Sans renier ses objectifs traditionnels - préserver la forêt et assurer une production économique durable au plus haut niveau la sylviculture doit répondre aux aspirations sociales de l'être humain dont le bien-être est le but final de toute l'activité.

Le forestier a donc un rôle important à jouer dans le domaine de la préservation de la nature à chaque niveau : primairement en stimulant la fonction polyvalente de la forêt en tant qu'élément important de l'environnement humain, secondairement en acceptant de coopérer étroitement avec des spécialistes d'autres disciplines.

Cette réorientation de la ligne de conduite et de la politique forestière exige une réflexion portant sur le profil professionnel du forestier de l'avenir.

Elle demande aussi une adaptation des techniques et des procédures actuelles, une réorganisation fondamentale de l'enseignement et de la recherche ainsi qu'une conception rénovée de l'organisation de l'administration forestière.

\section{BIBLOIGRAPHIE}

HASEL K. : Waldwirtschaft und Umwelt. Hamburg und Berlin, 1971.

JUTZI W. : Staubniederschlag und Schwefeldioxyd-Immissionen im Schweizerischen Mittelland. Bauztg. 86 (46) 1968.

KELLER TH. : Die Bedeutung des Waldes für den Umweitschutz. S.z.F. 122 (1I) 1971

LEIBUNDGUT H. : La Foresterie de l'An 2000. S.Z.F. 122 (11) 1971.

VAN MIEGROET, M. : Waldbau der Zukunft. Forstarchiv. 37 (2) 1967.

VAN MIEGROET, M. : De Positie van het Bos in Vlaanderen. Groene Band. I $(1 / 2) 1971$.

W.G.F.E.T, Stockholm 1971 . 


\section{SAMENVATTING}

\section{Bosbouw en Milieuproblematiek}

De bosbouw streeft een bestendig en veelzijdig bosgebruik na, dat onderhevig is an kwalitatieve en kwantitatieve variaties in ruimte en tijd.

In de geindustrialisecrde wereld grijpt hierbij een verplaatsing van de zwaartepunten plaats en wordt de bosbouw in toenemende mate gekonfrontecrd met het sociaal en ekonomisch alternatief.

Inzake milicubescherming zijn de volgende funkties van essenticel belang : de ruimte- of oppervlalctewerking, de welvaartswerking in de ruimste zin, de recreatieve opdracht en het vervullen van een opdracht inzake grondreserve.

Daarenboven kan de bosbouwer belangrijke hulp verstrekken bij de oplossing van problemen die betrekking hebben op de bescherming van de natuur, de landschapsopbouw en de ruimtelijke ordening.

Dit veronderstelt evenwel sociale motivering en een grondige wijziging van het beroepsprofiel van de bosbouwingenicur, die in de aard van zijn vorming moeten terug te vinden zijn.

\section{ZUSAMMENFASSUNG}

\section{Forstwirtschaft und Umweltsproblematik}

Die Forstwirtschaft strebt die ständige und möglichst vielseitige Waldbenutzung an. Diese ist qualitativer und quantitativer Variationen in Raum und Zeit unterliegen.

In der industrialisierten Welt findet eine ständige Verschiebung der Schwergewichte statt und wird die Forstwirtschaft in zunehmendem Ausmas mit einer alternativen Wahl zwischen ökonomischen und sozialen Objektiven konfronticrt.

In Fragen der Umweltsproblematik sind nachstende Waldfunktionen erster Wichtigkeit: Die Raumwirkung, die allgemeine Wohlfahrtswirkung, die rekreative Aufgabe und die Bildung einer Bodenreserve.

Dic Forstwirtschaft hat ausserdem eine bedeutende Rolle zu erfülen im Gebiet des Naturschutzes, der Landschaftgestaltung und der Raumordnung.

Diese Nebenaufgaben erforderen eine soziale Motivierung und eine gründliche Umstellung des Berulsprofils, dass bei der Ausbildung zum Ausdruck gebracht werde muss.

\section{SUMMARY}

\section{Forestry and the Ecological Grisis}

The principal aim of forestry is to obtain an optimal, continuous and multiple use of the forest. Actually forest use is characterized by qualitative and quantitative variations in time and space.

In the industrialized countries a continuous deplacement of the centres of gravity is taking place and forestry is confronted with an alternative choice between economic and social objectives.

As far as environmental problems are concerned the essential functions of the forest are: the physical presence, the protection of agricultural land and living space, the recreative function and the building of a soil reserve.

The forester can give considerable help in matters concerning protection of nature, landscaping and soil conservation.

The actual and multiple function of forestry requires a sound social motivation and a modification of the profile of the profession. These changes nust be taken into consideration in planning and organizing forestry education. 